\title{
"Contribución de la Ciencia Contable y Empresarial a la Gestión de la Responsabilidad Social de las Empresas y la protección del Medio Ambiente"
}

\section{"Contribution of Science and Business Accounting Management of Social Responsibility of Enterprises and Environmental Protection"}

\author{
Oswaldo J. Vásquez Cerna \\ Decano de la Facultad de ciencias Empresariales / Universidad Alas Peruanas \\ o_vasquez@uap.edu.pe
}

\section{RESUMEN}

La presente investigación tuvo como principal objetivo analizar y proponer las posibles funciones y aportaciones que puede realizar la contabilidad empresarial en la gestión del medio ambiente.

Se realizaron una encuesta vía correo electrónico a un total de 65 empresas del sector petrolero y químico y seis entrevistas estructuradas. Entre los resultados se observó que las empresas entrevistadas tienen implantado un sistema de gestión medioambiental y que está en constante fortalecimiento. Además se obtuvo que no existe una relación significativa entre la implantación de una política o un sistema de gestión medioambiental y la publicación de información financiera medioambiental.

Palabras clave: contabilidad ambiental, empresa, gestión de la responsabilidad social, protección, medio ambiente.

\section{ABSTRACT}

This research has as main objective to analyze and propose possible roles and contributions that can make business accounting in environmental management.

A survey via email to a total of 65 companies in the oil and chemicals sector six structured interviews. Among the results it found that the companies surveyed have implemented an environmental management system and is constantly strengthening. In addition it was found that there is no significant relationship between the implementation of a policy or environmental management system and environmental publishing financial information.

Keywords: environmental accounting, business management, social responsibility, environmental protection 


\section{Introducción}

La preservación del medio ambiente en las sociedades industriales es una de las preocupaciones existentes en la actualidad, explicitadas en la creación de diversos foros de análisis y estudio, entre los que destacan las Naciones Unidas (ONU), mediante la Comisión para el Medio Ambiente y Desarrollo (CNUMAD), la Unión Europea, a través de sus políticas y programas medioambientales, o el establecimiento de ministerios de medio ambiente en muchos de los países desarrollados y en desarrollo, con capacidad de toma de decisiones relevantes en este ámbito. Esta inquietud se centra especialmente en la actividad económica desarrollada por las empresas industriales, que constituyen una de las principales fuentes de la crisis medioambiental existente en el planeta en las últimas décadas. En este sentido, se está generando una presión creciente sobre las actividades empresariales, con el fin de que se adopten las medidas oportunas tendentes a evitar un mayor deterioro del entorno natural. Esta presión se está traduciendo en una mayor sensibilidad medioambiental por parte de los gestores de las compañías, que a su vez puede traducirse en la aparición de oportunidades competitivas para las mismas, a través de un comportamiento ecológico aceptado por la sociedad. En la puesta en práctica de esta conciencia ecológica, además de la función de dirección correspondiente a los gerentes, es importante la tarea de otros profesionales que operan en la empresa, entre los que tradicionalmente han destacado ingenieros, químicos, biólogos y otras profesiones científicotécnicas. Así, existe una amplia investigación y actividad por parte de estas disciplinas que se han involucrado desde hace años en la conservación de su entorno y han colaborado en su aplicación, tanto en el ámbito macroeconómico, como a nivel microeconómico o empresarial. Sin embargo, los expertos no han aprovechado, hasta la fecha, este nuevo campo de acción para desarrollar su ámbito de actuación y contribuir a su desarrollo junto al resto de disciplinas. Por tanto, los profesionales de la contabilidad, como principales analistas de la economía empresarial, tienen el reto y la oportunidad de asumir un papel relevante en la conservación del entorno, ya que poseen una serie de capacidades que les debe permitir ofrecer una importante colaboración; en este sentido, en las últimas décadas, la doctrina contable y empresarial ha abordado la investigación del tratamiento de diversos problemas sociales, entre los que destaca el medio ambiente. Algunos organismos internacionales, como la Organización de las Naciones Unidas (ONU) y la Unión Europea (UE), han creado grupos de trabajo sobre administración y medio ambiente para analizar su problemática.

\section{Objetivos del estudio}

- Analizar y proponer las posibles funciones y aportaciones que puede realizar la contabilidad empresarial en la gestión del medio ambiente, fundamentalmente en el ámbito de la responsabilidad social de la empresa y la protección del medio ambiente".

- Analizar la responsabilidad que debe asumir la empresa, como sistema informativo de la empresa, en la consideración de los distintos aspectos sociales que le afectan, entre los que la protección del medio ambiente se presenta como uno de los de mayor importancia, junto a los recursos humanos y otros valores éticos.

- Estudiar la forma en que los profesionales de la contabilidad pueden cumplir con la responsabilidad medioambiental de la empresa, en la parte que les corresponde; determinando las funciones que pueden desarrollar en la internalización de la variable ambiental en los procesos de decisión de la empresa.

- Conocer el grado de implantación de la gestión medioambiental en la práctica real de las empresas, el papel desempeñado por la administración en dicha gestión, así como las funciones realizadas por los expertos en el tratamiento de aspectos medioambientales, tanto en el ámbito de gestión, como en el de la información publicada.

\section{Material y método}

Participaron finalmente 65 empresas quienes respondieron los cuestionarios suministrados, además de 6 empresas a los cuales se les aplicó las entrevistas estructuradas. El análisis de resultados se desarrolló de forma paralela y complementaria entre los datos de la encuesta por correo y los de las entrevistas. En este sentido, la encuesta es analizada mediante un análisis descriptivo que se completa con algunos contrastes estadísticos, mientras que con los datos de la entrevista se realiza un análisis exploratorio y descriptivo debido a que el número de las mismas es pequeño y la información de difícil parametrización para su proceso matemático. 


\section{Contabilidad ambiental}

Mautz (1963, p. 319), que indica que la contabilidad trata sobre las empresas que son grupos sociales, se refiere a transacciones y eventos económicos que tienen consecuencias sociales y también influye en las relaciones sociales. Además, genera conocimientos de utilidad significativa para los seres humanos ocupados en actividades con implicaciones sociales. Por todo ello considera que la contabilidad es una ciencia social. Tal como indica García Echevarría (1982, p. 29), la responsabilidad social de la empresa así como la presentación de cuentas sociales, están directamente relacionadas con dichas interrelaciones. Por ello, la asunción de mayores responsabilidades sociales por parte de la empresa, puede llegar a ser un instrumento con gran capacidad de influencia sobre la sociedad. La primera fase de la responsabilidad empresarial, como indica Orr (1982), da origen a expresiones como apoyo empresarial, filantropía empresarial y participación voluntaria por parte de las empresas en la comunidad.

Como indica Garrido (1980), empresa, gobierno y sociedad en general se dan cita en esta problemática y se interrelacionan de tal forma, que todos ellos han variado sus roles originales y modificado sus esferas de actuación y área de poder, creándose un marco social en el que las decisiones de los distintos participantes no son siempre autónomas, pues en alguna medida vienen influidas, cuando no impuestas, por otros grupos sociales.

Por otro lado, pueden distinguirse diversos niveles de responsabilidad social por parte de la empresa, que según Hargreaves y Dauman (1975) se divide en:

\section{- Responsabilidades básicas \\ - Responsabilidades organizacionales \\ - Responsabilidades sociales}

AECA (1996, p. 38) se pronuncia al referirse a la contabilidad de gestión medioambiental, aduciendo que se refiere a la propia contabilidad de gestión a través de la cual se registra, valora, racionaliza y controla la proyección medioambiental de la empresa. La contabilidad social y medioambiental es definida por Gray et alt. (1987, p. ix) con el sentido de presentación de información a grupos interesados y a la sociedad en general. Así, consideran que es el proceso de comunicar los efectos sobre el entorno causados por las actividades económicas de las organizaciones. Los costos medioambientales relacionados de manera directa $\mathrm{o}$ indirecta con beneficios que se han producido en el ejercicio corriente, deben ser aplicados a dicho ejercicio como gastos. Además, aquellos costos medioambientales que son considerados costos corrientes o pérdidas, deben ser tratados igualmente como gastos del ejercicio (CICA, 1993, pp.19-20).

\section{Gestión ambiental en el Perú}

Un documento elaborado por el Ministerio del Ambiente (2012; pág.76) consideran la gestión ambiental como un:

Conjunto estructurado de principios, normas técnicas, procesos y actividades, orientado a administrar los intereses, expectativas y recursos relacionados con los objetivos de la política ambiental y alcanzar así, una mejor calidad de vida y el desarrollo integral de la población, el desarrollo sostenible de las actividades económicas y la conservación del patrimonio ambiental y natural del país.

La gestión ambiental tiene un carácter esencialmente transectorial lo que implica que la actuación de las diferentes autoridades públicas con competencias y responsabilidades ambientales se debe orientar, integrar, estructurar, coordinar y supervisar.

El Sistema Nacional de Gestión Ambiental (SNGA) es la organización integral de la gestión ambiental territorial y funcional, en materia ambiental y de los recursos naturales, teniendo en cuenta las competencias en biodiversidad, cambio climático, manejo de suelos y otros relacionados a los mismos.

El SNGA permite articular e interrelacionar el ejercicio de las funciones ambientales a cargo de las entidades públicas del nivel nacional, regional y local, los cuales implementan instrumentos de gestión ambiental para orientar el mejor desempeño de todas las actividades humanas y productivas regidas por su respectivo marco legal, los que permiten el cumplimiento de la Política Nacional del Ambiente. (Ministerio del Ambiente, 2013; pág. 9)

La finalidad del SNGA es orientar, integrar, coordinar, supervisar, evaluar y garantizar tanto la aplicación de los instrumentos de gestión ambiental, como políticas, planes, programas y acciones destinados a la protección del ambiente y a la conservación, así como al aprovechamiento sostenible de los recursos naturales. 


\section{Resultados y Discusión}

Cuadro $N^{\circ} 1$ : Importancia de la contabilidad en la gestión medioambiental

\begin{tabular}{|c|c|c|}
\hline Detalle & $\begin{array}{c}\mathbf{N}^{\mathbf{o}} \text { de } \\
\text { empresas }\end{array}$ & $\boldsymbol{\%}$ \\
\hline Papel relevante & 24 & 36,92 \\
\hline No desempeñan ningún papel o es irrelevante & 32 & 49,23 \\
\hline No contestan & 9 & 13,85 \\
\hline
\end{tabular}

Fuente: Elaboración propia

Podemos deducir que las empresas, en general, no consideran importante que la contabilidad participe en la gestión medioambiental, aunque los porcentajes indican una tendencia a un incremento en la importancia de la misma. Esto puede ser debido a que no se considera que los profesionales de la contabilidad puedan realizar tareas distintas de las tradicionales sujetas al ámbito puramente financiero.

Cuadro $N^{\circ}$ 2: El tamaño de la entidad

\begin{tabular}{|l|l|l|l|l|}
\hline & \multicolumn{2}{|l|}{ IFCO } & \multicolumn{2}{l|}{ IGMA } \\
\hline & U & Significatividad & U & Significatividad \\
\hline Grupo 1 y 2 & 194.5 & 0,290 & 231 & 0,834 \\
\hline Grupo 1 y 3 & 284 & 0,983 & 260.5 & 0,611 \\
\hline Grupo 2 y 3 & 118.5 & 0,263 & 140 & 0,686 \\
\hline
\end{tabular}

Fuente. Elaboración propia

Como conclusión general podemos decir que el tamaño de las entidades no es un atributo diferenciador entre las empresas de los sectores analizados respecto de su comportamiento medioambiental medido por la gestión y actuaciones ecológicas (IGMA). Tampoco es un factor distintivo para observar la importancia de las diferentes funciones y papeles desarrollados por la contabilidad empresarial en la gestión y actividades medioambientales.

\section{El sector de actividad de las entidades}

\begin{tabular}{|l|r|r|}
\hline & \multicolumn{1}{|c|}{ IFCO } & \multicolumn{1}{c|}{ IGMA } \\
\hline U de & 159,000 & 123,500 \\
Mann-Whitney & & \\
W de & 195,000 & 159,500 \\
Wilcoxon & $-1,392$ & $-2,109$ \\
$Z$ &, 164 &, 035 \\
$\begin{array}{l}\text { Sig. asintót. } \\
\text { (bilateral) }\end{array}$ & \\
\hline
\end{tabular}

Con un nivel de significación del 0,05 no se puede rechazar la hipótesis nula de que la función contable desempeñada en ambos sectores, medida por el indicador IFCO, es homogénea. Únicamente podríamos admitir que la diferencia es importante para el nivel de significatividad de 0,164 , lo cual no es un grado muy relevante. En definitiva, las empresas químicas tienen más desarrollada la gestión medioambiental que las petrolíferas, pero la importancia de las funciones asignadas a la contabilidad en la misma es similar entre ambas. 


\section{La política y gestión medioambiental}

\begin{tabular}{|l|r|r|}
\hline & \multicolumn{1}{|c|}{ IFCO } & \multicolumn{1}{c|}{ IGMA } \\
\hline U de & 195,000 & 11,000 \\
Mann-Whitney & & \\
W de & 250,000 & 66,000 \\
Wilcoxon & $-1,469$ & $-4,851$ \\
$Z$ &, 142 &, 000 \\
\hline $\begin{array}{l}\text { Sig. asintót. } \\
\text { (bilateral) }\end{array}$ & & \\
\hline
\end{tabular}

Existe un alto nivel de significación $(\mathrm{p}=0,000)$ para rechazar la hipótesis nula de comportamiento homogéneo entre las dos submuestras para el indicador IGMA. Esto indica, que las entidades con una actuación medioambiental definida mediante una política o instrumentada en un sistema de gestión, desarrollan actividades ecológicas en mayor medida y relevancia que las que no tienen sus actuaciones sistematizadas y actúan mediante operaciones puntuales. Por otro lado los profesionales de la contabilidad no han desarrollado funciones de relevancia en la gestión medioambiental implantada en las empresas analizadas, puesto que no se diferencian de las entidades que no han implantado tal gestión.

\section{Certificación del sistema de gestión medioambiental}

\begin{tabular}{|l|r|r|}
\hline & \multicolumn{1}{|c|}{ IFCO } & \multicolumn{1}{|c|}{ IGMA } \\
\hline U de & 443,000 & 207,000 \\
Mann-Whitney & & \\
$\begin{array}{l}\text { W de } \\
\text { Wilcoxon }\end{array}$ & 1478,000 & 1242,000 \\
$Z$ &,- 100 & $-3,490$ \\
$\begin{array}{l}\text { Sig. asintót. } \\
\text { (bilateral) }\end{array}$ &, 920 &, 000 \\
\hline
\end{tabular}

Llegamos a la conclusión de que existen diferencias significativas en cuento a la valoración obtenida por cada grupo respecto al sistema de gestión $(\mathrm{p}=0,000)$, mientras que no aparecen respecto a las funciones de la contabilidad desempeñadas por ambas submuestras. Obviamente, tal como indican las ordenaciones promedio, el grupo que mejor se comporta en cuanto a la gestión y comportamiento ecológico es el que se encuentra certificado.

\section{Conclusiones}

- En general, las empresas analizadas consideran importante la implantación de una gestión medioambiental, motivadas principalmente por presiones internas, como es el propio compromiso, y por presiones externas, fundamentalmente la normativa legal. Esta relevancia se traduce en el elevado nivel jerárquico de los responsables medioambientales y en el creciente número de certificaciones de los sistemas de gestión.

- Las entidades han asumido el reto ecológico desde una perspectiva de desarrollo sostenible, ya que sus actuaciones medioambientales son de prevención y reducción de daños, o de cumplimiento de legislación y, además, los logros obtenidos no son valorados en función de su vertiente financiera sino ética o ecológica.

- La teoría de los partícipes o Stakeholders parece ser explicativa del comportamiento informativo de las empresas en relación con sus actuaciones medioambientales, debido a que se persigue conseguir el apoyo de las diversas personas sociales interesadas en la actividad de las empresas, sin el cual éstas no podrían sobrevivir. Los resultados obtenidos o esperados de tal publicación están relacionados con la imagen y la aceptación social de la entidad, lo que viene relacionado con dicha teoría.

- Los profesionales de la contabilidad tienen importantes retos que afrontar en la gestión medioambiental mediante su colaboración en varias funciones esenciales de la misma:

1) Los sistemas de control, instrumentados en las auditorías medioambientales, en las que pueden aportar sus conocimientos de las auditorías financieras.

2) La documentación y presentación de información del sistema de gestión, debido a su función de sistema informativo empresarial.

- Las funciones desarrolladas por la contabilidad en la gestión medioambiental se han limitado a las tradicionales, siendo su principal aportación la elaboración y control de los presupuestos medioambientales y el tratamiento de los costos, aunque se cree que su papel debería ser mayor en la presentación de información ambiental. Esto confirma nuestra opinión de que los profesionales contables deben aplicar sus técnicas, 
conocimientos y experiencia al tratamiento de los aspectos medioambientales y la presentación de información, para lo cual se hace necesario trabajar junto a profesionales de otras áreas de conocimiento.

- La contabilidad de gestión no utiliza técnicas específicas para el tratamiento de los costos medioambientales, lo que oculta su relevancia entre los demás costos indirectos y provoca una asignación incorrecta.

- Se considera que deberían establecerse los mecanismos que permitan su tratamiento diferenciado, con el fin de poder conocer su relevancia. La asignación específica y la utilización de técnicas como el análisis del ciclo de vida de los productos y el sistema de costos ABC pueden ser de utilidad en la gestión de los mismos.

- En la valoración de los costos medioambientales deben aplicarse los principios contables generalmente aceptados, mientras que las metodologías propuestas por la economía ambiental no son aplicables al ámbito empresarial.

- Aunque distintas partidas contables se ven afectadas de forma notable por los aspectos medioambientales, no suelen tratarse o presentarse de forma separada por parte de la contabilidad financiera. En este sentido, el departamento de contabilidad debería colaborar más activamente mediante la coordinación de las partidas financieras que se deberían presentar y el apoyo en la creación de sistemas informativos más eficientes.

- El tamaño de las entidades y la nacionalidad de la matriz no son factores diferenciadores respecto al grado de implicación de las empresas en la protección del entorno. Sin embargo, la existencia de un sistema de gestión medioambiental implantado y la certificación del mismo, o la pertenencia a uno de los dos sectores analizados sí que lo son.

- Ninguno de los atributos analizados diferencian a las compañías respecto a la importancia del papel desempeñado por los profesionales de la contabilidad empresarial en la gestión medioambiental. Este hecho constituye una clara evidencia de que la profesión contable no interviene de forma activa en el desempeño de dicha gestión.

\section{Referencias bibliográficas}

[1] Asociación Española de Contabilidad y Administración de Empresas (AECA) (1996): Documento sobre principios de contabilidad de gestión $\mathrm{n}^{\circ} 13$, «Contabilidad de Gestión Medioambiental». AECA, Madrid.

[2] CANADIAN INSTITUTE OF CHARTERED ACCOUNTANTS (CICA) (1993): Environmental Costs and Liabilities: Accounting and Financial Reporting Issues. CICA, Toronto.

[3] GARCÍA ECHEVARRIA, S. (1982): Responsabilidad social y balance social de la empresa. Fundación MAPFRE, Madrid.

[4] GARRIDO, S. (1980): «La responsabilidad social de la empresa y su medición». Economía Industrial, no 196 , abril, pp. 1022.

[5] GRAY, R.; OWEN, D. y MAUNDERS, K. (1987): Corporate Social Reporting: Accounting and Accountability. Prentice Hall, London.

[6] HARGREAVES, J. y DAUMAN, J. (1975): Business Survival and Social Change. ABP, Londres.

[7] MAUTZ, R. K. (1963): «Accounting as a Social Science». The Accounting Review, abril, p. 319.

[8] MINISTERIO DEL AMBIENTE (2012): Glosario de términos para la gestión ambiental peruana. MINAM: Lima

[9] MISTERIO DEL AMBIENTE (2013): Sistema Nacional de Gestión Ambiental. MINAM: Lima

[10] ORR, D.J. (1982): «La responsabilidad social. Una nueva línea de negocios». Banco de Bilbao, pp. 524-526. 